\title{
Percentual de postura de codornas europeias alimentadas com diferentes níveis de lisina utilizando regressão beta
}

\author{
Silva, M.C.S.; Lucena, L.R.R. ${ }^{\circledR}$; Holanda, M.A.C. e Holanda, M.C.R.
}

Universidade Federal Rural de Pernambuco. Brasil.

\section{PALAVRAS CHAVE ADICIONAIS}

Producão de ovo.

Modelagem.

Ajuste.

Aves.

Dietas.

\section{RESUMO}

A criação de codornas para a produção de ovos está se expandindo nos últimos anos, sendo uma boa alternativa para qualidade nutricional da população. A grande procura por ovos de alta qualidade envolve o processo de produção com relevância nas características de peso, cor, integridade da casca e uniformidade. Para explicar o percentual de postura de codornas europeias alimentadas com diferentes níveis de lisina foi utilizado o modelo de regressão beta. $O$ modelo de regressão beta é utilizado para modelar taxas e proporções como no caso do percentual de postura das cordonas. Após ajuste, o modelo apresentou poder de explicação de 96,61\%, e identificou como variáveis explicativas para o percentual de posturas das codornas nível de lisina inseridos na dieta, número de ovos por aves alojadas, conversão alimentar e consumo de lisina. Observou-se que a cada incremento do nível de lisina espera-se em média um aumento de $29,3 \%$ no percentual de postura, já a cada incremento do número de ovos por ave alojada esta taxa é de $56,5 \%$, enquanto que para cada incremento na conversão alimentar e no consumo de lisina, respectivamente espera-se um decréscimo de $0,3 \%$ no percentual de postura das codornas. $\bigcirc$ modelo de regressão beta se mostrou bastante eficaz quando comparado aos modelos empregados usualmente na modelagem do percentual de postura das codornas europeias.

\section{Percentage of laying of European quails fed with different lysine of levels using beta regression}

\section{SUMMARY}

The creation of quails for egg production is expanding in the last years, being a good alternative for nutritional quality of population. The great demand for high quality eggs involves the production process with relevance in the characteristics of weight, color, shell integrity and uniformity. To explain the percentage of laying of european quails fed with different lysine levels, the beta regression model was used. The beta regression model is used to model rates and proportions as in the case of posture of the birds. After adjustment, the model presented explanatory power of $96.61 \%$, and identified as explanatory variables for the percentage of postures of the quails lysine level inserted in the diet, number of eggs per birds housed, feed conversion and lysine consumption. It was observed that at each increment of lysine level, a $29.3 \%$ increase in the percentage of posture is expected, since at each increment of the number of eggs per bird housed this rate is $56.5 \%$, while for each increase in feed conversion and in lysine consumption respectively, a decrease of $0.3 \%$ in the percentage of laying of quails. The beta regression model was very effective when compared to models usually employed in modeling of the European quail laying percentage.

\section{INTRODUÇÃO}

A criação de codornas para a produção de carne e ovos está desenvolvendo de forma expressiva no Brasil, sendo uma boa alternativa para obtenção de produtos de alta qualidade nutricional para a população (Móri et al., 2005). A produção de ovos de excelente qualidade é um fator de grande relevância, uma vez essa qualidade determinará o sucesso do produto no mercado consumidor. A grande procura por ovos de alta qualidade envolve o processo de produção e distribuição com relevância às características de peso, cor, integridade da casca e uniformidade (Xavier et al., 2008). 
Estudos científicos ralatam a utilização de modelos de regressão linear e quadrático para avaliar o percentual de postura em codornas japonesas (Ribeiro et al., 2013; Nery et al., 2015; Lima et al., 2016). Lima et al. (2016) avaliando diferentes níveis de lisina na dieta de codornas japonesas verificaram nível ótimo de lisina de $1,18 \%$ para maximizar o percentual de postural em $64 \%$. Outros autores encontraram percentuais de postura mais altos (de 76,96 a 93,31\%), para níveis de lisina que variaram de 1,045 a 1,10\% (Oliveira et al., 1999; Pinto et al., 2003; Costa et al., 2008; Moura et al., 2008; Ribeiro et al., 2013; Nery et al.; 2015). O modelo de regressão linear e quadrático também foi avaliado em codornas europeias por Della-Flora et al. (2012) que verificaram percentual de postura de $86,65 \%$ para um nível ótimo de lisina de 1,1137\%, corroborando a afirmacão de que o percentual de postura aumenta quando codornas são alimentadas com níveis de lisina mais altos nas dietas.

A análise de regressão é uma técnica estatística utilizada para modelar, com base em um conjunto de informação, a relação entre a variável de interesse e uma ou mais variáveis explicativas. O modelo de regressão linear com distribuição normal é bastante utilizado em análises empíricas. No entanto, tal modelo torna-se inapropriado em situações em que a variável resposta é restrita ao intervalo $(0,1)$, como ocorre com taxas e proporções (Ferrari e Cribari Neto, 2004). O modelo de regressão em que a variável resposta apresenta valores no intervalo $(0,1)$ é denominado de modelo de regressão beta (Ferrari e Cribari Neto, 2004).

Os modelos de regressão beta também estão sendo aplicados para avaliação da taxa de postura de ovos em peixes. Quintero et al. (2011) avaliaram o conteúdo de proteína de ovos $\left(\mathrm{g} . \mathrm{kg}^{-1}\right)$ de peixe. Da mesma forma, Schlegel et al. (2012) avaliaram sucesso reprodutivo em ouriços, destacando-se a exigência de níveis mais altos em aminoácidos essenciais.

Nas ciências florestais estes modelos também são utilizados, destacando-se os trabalhos de Li et al. (2002) que modelaram o diâmetro e a altura de árvores conjuntamente; os de Korhonen et al. (2007) que avaliaram o percentual de cobertura do dossel; os de Ekleston et al. (2011) que avaliaram o percentual de cobertura dos arbustos e os de Laliberte et al. (2012) para avaliar a taxa de decomposição de lixo.

No intuito de encontrar uma função matemática para explicar o comportamento do percentual de postura das aves, foi utilizado o modelo de regressão beta para modelar o percentual de postura de codornas Coturnix Coturnix levando em consideração o número de ovos por aves alojadas, o peso dos ovos, o consumo de ração, a conversão alimentar, entre outras variáveis.

\section{MATERIAL E MÉTODOS}

A pesquisa foi na Universidade Federal Rural de Pernambuco, Unidade Acadêmica de Serra Talhada (UAST), localizada nas coordenadas geográficas em latitude $07^{\circ} 57^{\prime} 01^{\prime \prime}$ sul e longitude $38^{\circ} 17^{\prime} 53^{\prime \prime}$ oeste, com uma altitude de aproximadamente $429 \mathrm{~m}$, localizada no município de Serra Talhada, Microrregião do Sertão do Pajeú, Mesorregião do Sertão Pernambucano. O clima local é do tipo BSwh', de acordo com a classificação de Koppen, muito quente e semiárido. A precipitação pluvial média anual é de $876 \mathrm{~mm}$ e a temperatura do ar média anual é de $27^{\circ} \mathrm{C}$ (Lucena, 2016).

Foram utilizadas 175 codornas fêmeas da linhagem europeia (Coturnix coturnix sp) com 65 dias de idade, distribuídas em um delineamento inteiramente casualizado, com cinco tratamentos e cinco repetições por tratamento, alojadas em 25 gaiolas metálicas, cada uma representando uma parcela experimental, com sete aves por parcela. Cada gaiola foi provida de bebedouro tipo niple e comedouro tipo calha, e bica de coleta e apanha de ovos. Água e ração foram providas ad libitum.

Os tratamentos consistiram de cinco níveis crescentes $(1,117 ; 1,217 ; 1,317 ; 1,417$ e $1,517 \%)$ de lisina digestível na dieta e as rações foram formuladas de acordo com as recomendações de Rostagno et al. (2017), contendo $23 \%$ de proteína bruta e energia metabolizável aparente de $2900 \mathrm{kcal} / \mathrm{kg}$ com o nível inicial de lisina digestível de 1,117\% mantendo-se a relação ideal para os aminoácidos, metionina digestível, metionina + cistina digestível, treonina digestível e triptofano digestível, Tabela I.

O manejo foi realizado conforme as recomendações do Manual de Criação da Linhagem. A partir do sexagésimo quinto dia de vida as aves foram submetidas a um programa de luz de 17 horas de fotoperíodo controlado por um relógio automático (timer) e receberam as dietas experimentais de acordo com os tratamentos e os níveis de lisina das rações experimentais.

Foram acompanhados e anotados em fichas de controle o abastecimento e sobras de ração, para aferir o consumo de ração, ovos produzidos por parcela, produção média de ovos por ave/dia (\%), consumo de ração (g/ave/dia), peso dos ovos (g) e a conversão alimentar ( $g$ de ração por massa de ovo e por dúzia de ovos).

A coleta dos ovos foi realizada diariamente, pela manhã e à tarde, e a produção média de ovos foi obtida dividindo-se o total de ovos produzidos (ovos inteiros, quebrados, trincados e deformados) pelo número de aves viáveis de cada parcela. A conversão alimentar, dividindo-se o total de ração consumida pelo peso dos ovos produzidos, sendo expressa em gramas de ração por grama de ovo produzido. A conversão alimentar por dúzia de ovos foi obtida pelo produto entre o consumo médio de ração e a dúzia de ovos produzidos. Com o peso total e o número de ovos por parcela, foi calculado o peso médio dos ovos das parcelas. A viabilidade das aves foi calculada pela subtração do número de aves alojadas no início do experimento pela mortalidade, em valor percentual.

A relação entre explicar o percentual de postura das aves em função das variáveis: ovo por aves alojadas (OVO), peso do ovo (POVO), nível de lisina (NL), consumo de ração (CR), conversão alimentar por dúzia (CA), conversão alimentar por massa (CAM) e consumo de lisina (CL) foi estudada adotando o modelo de regressão beta como descrito abaixo. 


\begin{tabular}{|c|c|c|c|c|c|}
\hline \multirow[t]{2}{*}{ Ingredientes } & \multicolumn{5}{|c|}{ Níveis de lisina digestível (\%) } \\
\hline & 1,117 & 1,217 & 1,317 & 1,417 & 1,517 \\
\hline Milho moído (kg) & 43,366 & 43,366 & 43,366 & 43,366 & 43,366 \\
\hline Farelo de soja (kg) & 40,200 & 40,200 & 40,200 & 40,200 & 40,200 \\
\hline Fosfato bicálcico (kg) & 1,590 & 1,590 & 1,590 & 1,590 & 1,590 \\
\hline Calcário calcítico (kg) & 7,480 & 7,480 & 7,480 & 7,480 & 7,480 \\
\hline Sal comum (kg) & 0,424 & 0,424 & 0,424 & 0,424 & 0,424 \\
\hline DL-metionina (kg) & 0,160 & 0,160 & 0,160 & 0,160 & 0,160 \\
\hline L-lisina HCl (kg) & 0,000 & 0,100 & 0,200 & 0,300 & 0,400 \\
\hline Óleo de soja (kg) & 5,310 & 5,400 & 5,450 & 5,55 & 5,600 \\
\hline Amido (kg) & 1,270 & 1,170 & 1,070 & 0,970 & 0,870 \\
\hline Premix vitamínico e mineral $(\mathrm{kg})$ & 0,200 & 0,200 & 0,200 & 0,200 & 0,200 \\
\hline Total & 100 & 100 & 100 & 100 & 100 \\
\hline \multicolumn{6}{|l|}{ Composição química das dietas } \\
\hline Amido (\%) & 32,336 & 32,280 & 32,250 & 32,140 & 32,130 \\
\hline Arginina digestível (\%) & 1,444 & 1,443 & 1,440 & 1,450 & 1,450 \\
\hline Cálcio (\%) & 3,394 & 3,394 & 3,390 & 3,390 & 3,390 \\
\hline Cloro (\%) & 0,042 & 0,042 & 0,040 & 0,040 & 0,040 \\
\hline Energia metabolizável (Kcal/kg) & 2.900 & 2.900 & 2.900 & 2.900 & 2.900 \\
\hline Fenilalanina digestível (\%) & 1,018 & 1,018 & 1,018 & 1,018 & 1,018 \\
\hline Fenilalanina+Tirosina digestível (\%) & 1,715 & 1,714 & 1,710 & 1,713 & 1,714 \\
\hline Fibra bruta (\%) & 2,932 & 2,931 & 2,931 & 2,931 & 2,931 \\
\hline Fósforo disponível (\%) & 0,401 & 0,401 & 0,401 & 0,401 & 0,401 \\
\hline Gordura (\%) & 2,248 & 2,245 & 2,240 & 2,240 & 2,250 \\
\hline Histidina digestível (\%) & 0,551 & 0,551 & 0,551 & 0,551 & 0,551 \\
\hline Isoleucina digestível (\%) & 0,885 & 0,885 & 0,890 & 0,890 & 0,890 \\
\hline Leucina digestível (\%) & 1,719 & 1,718 & 1,720 & 1,720 & 1,720 \\
\hline Lisina digestível (\%) & 1,117 & 1,217 & 1,317 & 1,417 & 1,517 \\
\hline Metionina digestível (\%) & 0,461 & 0,461 & 0,461 & 0,460 & 0,460 \\
\hline Metionina+Cistina digestível (\%) & 0,590 & 0,590 & 0,590 & 0,590 & 0,590 \\
\hline Pótassio (\%) & 0,858 & 0,858 & 0,858 & 0,858 & 0,858 \\
\hline Proteína bruta (\%) & 21,830 & 21,830 & 21,830 & 21,830 & 21,830 \\
\hline Sódio (\%) & 0,230 & 0,230 & 0,230 & 0,230 & 0,230 \\
\hline Treonina digestível (\%) & 0,749 & 0,749 & 0,749 & 0,749 & 0,749 \\
\hline Triptofano digestível (\%) & 0,251 & 0,251 & 0,251 & 0,251 & 0,251 \\
\hline
\end{tabular}

Premix vitamínico e mineral por kg de ração: vit. A 3.750 .000 Ul; vit. D3, 750.000 Ul; vit. E 7500mg; vit K3, 1.000mg; vit. B1, 750mg; vit. B2, $1.500 \mathrm{mg}$; vit. B6, $1500 \mathrm{mg}$; vit. B12, $7.500 \mathrm{mcg}$; vit. C $12.500 \mathrm{mg}$, biotina $30 \mathrm{mg}$, niacina $10.000 \mathrm{mg}$, ácido fólico 375 ; Ácido pantotênico $3.750 \mathrm{mg}$; colina $10.000 \mathrm{mg}$, metionina $400.000 \mathrm{mg}$, selênio $45 \mathrm{mg}$; iodo $175 \mathrm{mg}$; ferro $12.525 \mathrm{mg}$; cobre 2.500mg; manganês, $19.500 \mathrm{mg}$; zinco 13.750mg; prom. Prod 15.000mg, coccidiostático $10.000 \mathrm{mg}$, antioxidante (B.H.T) 500mg.

Ferrari e Cribari Neto (2004) propuseram a classe de modelos de regressão beta, em que a variável resposta (Y) possui distribuição beta. A estrutura e procedimentos inferenciais do modelo são similares aos dos modelos lineares generalizados (McCullagh e Nelder, 1989).

Seja Y uma vaiável aleatória com distribuição Beta $(p, q)$, então definimos sua função de densidade de probabilidade por:

$$
f(y ; p, q)=\frac{\Gamma(p+q)}{\Gamma(p) \Gamma(q)} y^{p-1}(1-y)^{q-1}
$$

em que, y é a variável continua no intervalo $(0,1)$, p $>0$ e q $>0$ são parâmetros da função de densidade de probabilidade e $\left.\Gamma^{*}\right)$ é a função gamma, definida por:

$$
\Gamma(\mathrm{p})=\int_{0}^{\infty} \mathrm{y}^{\mathrm{p}-1} \mathrm{e}^{-\mathrm{y}} \mathrm{dy}
$$

No modelo de regressão proposto em Ferrari e Cribari-Neto (2004) é utilizada uma reparametrização 
da densidade de probabilidade da Beta, sendo $\mu=p /$ $(p+q)$ e $\emptyset=p+q$, isto é, $p=\mu \emptyset$ e $q=(1-\mu) \emptyset$, logo a função de densidade fica expressa por:

$$
\mathrm{f}(\mathrm{y} ; \mu, \emptyset)=\frac{\Gamma(\emptyset)}{\Gamma(\mu \emptyset) \Gamma((1-\mu) \emptyset)} \mathrm{y}^{\mu \emptyset-1}(1-\mathrm{y})^{(1-\mu) \emptyset-1}
$$
por:

logo, a estrutura do modelo de regressão é dada

$$
g\left(\mu_{\mathrm{i}}\right)=\eta_{\mathrm{i}}=\beta_{0}+\beta_{1} \mathrm{X}_{\mathrm{i} 1}+\beta_{2} \mathrm{X}_{\mathrm{i} 2}+\cdots+\beta_{\mathrm{k}} \mathrm{x}_{\mathrm{ik}}
$$

em que, ๆé o preditor linear, $\beta=\left(\beta_{0}, \beta_{1}, \beta_{2}, \ldots, \beta_{\mathrm{k}}\right)$ é o vetor de parâmetros desconhecidos e $\mathrm{X}_{\mathrm{i} 1}, \mathrm{X}_{\mathrm{i} 2}, \ldots, \mathrm{X}_{\mathrm{ik}}$ são as observações de $\mathrm{k}$ variáveis independentes conhecidas $(k<n)$ e $i=1,2, \ldots, n$. No modelo de regressão beta, pode-se escolher diferentes funções de ligação como, por exemplo, a função logit:

$$
g(\mu)=\log \left(\frac{\mu}{1-\mu}\right)
$$

onde, a média da variável $y_{i}$ pode ser escrita em função das variáveis explicativas através da seguinte expressão:

$$
\mu_{\mathrm{i}}=\frac{\exp ^{\left(\beta_{0}+\beta_{1} \mathrm{x}_{\mathrm{i} 1}+\beta_{2} \mathrm{x}_{\mathrm{i} 2}+\cdots+\beta_{\mathrm{k}} \mathrm{x}_{\mathrm{ik}}\right)}}{1+\exp ^{\left(\beta_{0}+\beta_{1} \mathrm{x}_{\mathrm{i} 1}+\beta_{2} \mathrm{x}_{\mathrm{i} 2}+\cdots+\beta_{\mathrm{k}} \mathrm{x}_{\mathrm{ik}}\right)}}
$$

em que, $0<\mu_{i}<1$. A estimação dos coeficientes é obtida pela maximização da função de log-verossimil- hança, e os ajustes avaliados pelo pseudo coeficiente de determinação do modelo (pseudo- ${ }^{2}$ ) e pela soma de quadrado de resíduo (SQR) (Ferrari e Cribari-Neto, 2004).

\section{RESULTADOS E DISCUSSÃO}

Avaliando o percentual de postura nota-se que, em média, as aves apresentaram uma produção de $78 \pm 0,06 \%$ com mínimo de $64 \%$ e máximo de $87 \%$. O

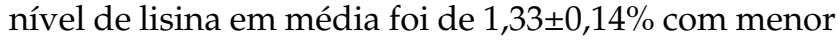
valor de $1,13 \%$ e maior de $1,53 \%$, o número de ovos por ave alojada foi em média 5,35 $\pm 0,48$ apresentando menor valor de 4,28 e maior valor 6,10. Já a média do peso dos ovos foi de $1083,8 \pm 348,81 \mathrm{~g}$ com mínimo valor de 374 g e máximo de 1756 g, a média do consumo de ração foi de $3559,12 \pm 873,67 \mathrm{~g}$, enquanto que a média da conversão alimentar por dúzia e por massa foram de $467,96 \pm 40,44$ e 3,15 $\pm 0,27$, respectivamente, já a média do consumo de lisina foi de $47,45 \pm 13,45 \mathrm{~g}$ com menor valor de 15,89 g e máximo de 83,37 g, Tabela II.

Na Tabela III verifica-se o grau de correlação das variáveis, nota-se que o nível de lisina não se correlaciona com o percentual de postura $(\mathrm{r}=-0,473)$, assim como para o consumo de lisina $(r=0,498)$. A conversão alimentar por dúzia e a conversão alimentar por massa apresentam correlação negativa com o percentual de postura das aves $(r=-0,990$ e e $r=-0,935$, respectivamente). Já a quantidade de ovos por ave alojada se correlaciona positivamente com o percentual de postura $(\mathrm{r}=0,951)$.

\begin{tabular}{|c|c|c|c|c|c|c|c|c|}
\hline & PP & NL & OVO & POVO & CR & CA & CAM & $\mathrm{CL}$ \\
\hline PP & 1,00 & -- & -- & -- & -- & -- & -- & -- \\
\hline NL & $-0,473$ & 1,00 & -- & -- & -- & -- & -- & -- \\
\hline OVO & $0,951^{*}$ & $-0,194$ & 1,00 & -- & -- & -- & -- & -- \\
\hline POVO & 0,414 & 0,116 & 0,484 & 1,00 & -- & -- & -- & -- \\
\hline CR & 0,416 & 0,179 & 0,469 & $0,918^{*}$ & 1,00 & -- & -- & -- \\
\hline CA & $-0,990^{*}$ & 0,220 & $-0,956^{*}$ & $-0,440$ & $-0,430$ & 1,00 & -- & -- \\
\hline CAM & $-0,935^{*}$ & $-0,090$ & $-0,890^{*}$ & $-0,361$ & $-0,289$ & $0,946^{*}$ & 1,00 & -- \\
\hline $\mathrm{CL}$ & 0,498 & 0,537 & 0,319 & $0,809^{*}$ & $0,922^{*}$ & $-0,305$ & $-0,228$ & 1,00 \\
\hline
\end{tabular}

Tabela II. Medidas descritivas das variáveis em estudo (Descriptive measures of the variables under study).

\begin{tabular}{lcccc}
\hline Variáveis & Min & Media & Desvio P. & Max \\
\hline Percentual de Postura (\%) & 0,64 & 0,78 & 0,06 & 0,87 \\
Nível de Lisina (\%) & 1,13 & 1,33 & 0,14 & 1,53 \\
Ovo/Ave (und.) & 4,28 & 5,35 & 0,48 & 6,10 \\
Peso do ovo (g) & 374,0 & 1083,8 & 348,81 & 1756,0 \\
Consumo de ração (g/ave/dia) & 1410,0 & 3559,12 & 873,67 & 5460,0 \\
Conversão Alimentar (g) & 412,76 & 467,96 & 40,44 & 564,0 \\
Conversão Alimentar por massa (g) & 2,90 & 3,15 & 0,27 & 3,77 \\
Consumo de Lisina (g) & 15,89 & 47,45 & 13,45 & 83,37 \\
\hline
\end{tabular}

Tabela III. Correlação entre as variáveis em estudo (Correlation between the study variables).

*estatisticamente significativo ao nível de $5 \%(p<0,05)$ 
Tabela IV. Estimativa dos parâmetros do modelo inicial para explicar o percentual de postura das codornas (Estimation of initial model parameters to explain the birds laying percentage).

\begin{tabular}{|c|c|c|c|c|}
\hline Parâmetros & Estimativa & Desvio padrão & Estatística do teste & $p$ valor \\
\hline Intercepto & 2,73000 & 1,21000 & 2,265 & 0,0235 \\
\hline NL & 0,11000 & 0,37000 & 0,294 & 0,7691 \\
\hline OVO & 0,21000 & 0,11000 & 1,966 & 0,0453 \\
\hline POVO & $-0,00003$ & 0,00011 & $-0,292$ & 0,7700 \\
\hline CR & $-0,00001$ & 0,00014 & $-0,109$ & 0,9129 \\
\hline $\mathrm{CA}$ & $-0,00630$ & 0,00180 & $-3,437$ & 0,0006 \\
\hline CAM & 0,10300 & 0,22100 & 0,468 & 0,6398 \\
\hline$C L$ & $-0,00051$ & 0,01460 & $-0,048$ & 0,9618 \\
\hline
\end{tabular}

Inicialmente foi proposto o seguinte modelo para explicar o comportamento do percentual de postura das aves:

$$
P P=\frac{\exp ^{\left(\beta_{0}+\beta_{1} N L+\beta_{2} 0 V 0+\beta_{2} \text { POVO }+\beta_{3} C R+\beta_{4} C A+\beta_{5} C A M+\beta_{6} C L\right)}}{1+\exp ^{\left(\beta_{0}+\beta_{1} N L+\beta_{2} 0 V 0+\beta_{2} \text { POV } 0+\beta_{3} C R+\beta_{4} C A+\beta_{5} C A M+\beta_{6} C L\right)}}
$$

Após estimativas dos parâmetros verifica-se na Tabela IV, que algumas variáveis como: nível de lisina, peso do ovo, consumo de ração, conversão alimentar por massa e consumo de lisina conjuntamente não foram significativas para explicar o percentual de postura das aves.

Através do processo de ajuste dos modelos (stepway) procederam-se novas estimativas dos parâmetros a fim de se encontrar o modelo que melhor explicase o percentual de postura das aves, ficando definido que o modelo que melhor explica o percentual de postura deve ter como variáveis o nível de lisina, a quantidade de ovos por ave alojada, a conversão alimentar e o consumo de lisina, Tabela V.

Após ajuste dos parâmetros, o modelo ficou definido pela seguinte expressão:

$$
P P=\frac{\exp ^{(0,257 \mathrm{NL}+0,4480 \mathrm{~V} 0-0,003 \mathrm{CA}-0,003 \mathrm{CL})}}{1+\exp ^{(0,257 \mathrm{NL}+0,4480 \mathrm{~V} 0-0,003 \mathrm{CA}-0,003 \mathrm{CL})}}
$$

O modelo ajustado apresentou elevado coeficiente de determinação do modelo (pseudo- $\mathrm{R}^{2}=96,61 \%$ ) e baixa soma de quadrados dos resíduos (SQR=0,0044), medidas estas que qualificam o modelo como adequado. Através do modelo ajustado nota-se que a cada incremento do nível de lisina estima-se, em média, um aumento de $29,3 \%$ no percentual de postura. No entanto, para o número de ovos por ave alojada este aumento é estimado em $56,5 \%$, enquanto que para cada incremento na conversão alimentar e no consumo de lisina, respectivamente, espera-se um decréscimo de $0,3 \%$ no percentual de postura das aves.

Sabe-se que, quando a lisina é suprida de forma marginal, ou seja, deficientemente, o ganho de peso e a conversão alimentar podem ser visivelmente influenciados. A produção pode ser ainda mais afetada, pois as rações para codornas ainda são formuladas com base em resultados obtidos de pesquisas realizadas em outros países, como os preditos pelo National Research Council (NRC, 1994) e, portanto, pouco condizentes com a realiadade das condições brasileiras.

Mesmo sendo o segundo aminoácido mais limitante para aves, a lisina tem função especial, participando na deposição de proteína corporal e síntese de carnitina, que atua no transporte de ácidos graxos para a $\beta$-oxidação na mitocôndria. Em contapartida, o excesso de lisina também pode aumentar o custo metabólico por provocar antagonismo com outros aminoácidos, como a arginina, que disputa o mesmo sítio de absorção (Barreto et al., 2006).

Nas Figuras 1 a), b), c) e d), estão dispostos os valores observados do percentual de postura e os ajustados pelo modelo, nota-se que para todos os gráficos os valores estimados pelo modelo estão bem próximos aos valores observados, garantindo assim uma boa qualidade do ajuste do modelo.

Resultados obtidos neste estudo são superiores aos verificados por Della-Flora et al. (2012) que explicaram o percentual de postura de codornas europeias através do modelo quadrático com poder de explicação de $34 \%$ para um nível ótimo de lisina de $1,11 \%$, enquanto Ribeiro et al. (2003) apresentaram nos modelos quadrá-

Tabela V. Estimativa dos parâmetros do modelo ajustado para explicar o percentual de postura das codornas (Estimation of parameters of adjusted model to explain the birds laying percentage).

\begin{tabular}{lcccc}
\hline Parâmetros & Estimativa & Desvio padrão & Estatística do teste & $p$-valor \\
\hline NL & 0,257 & 0,1310 & 1,967 & 0,0492 \\
OVO & 0,448 & 0,0210 & 21,326 & $<0,0001$ \\
CA & $-0,003$ & 0,0003 & $-10,224$ & $<0,0001$ \\
CL & $-0,003$ & 0,0016 & $-5,823$ & 0,0383 \\
\hline
\end{tabular}



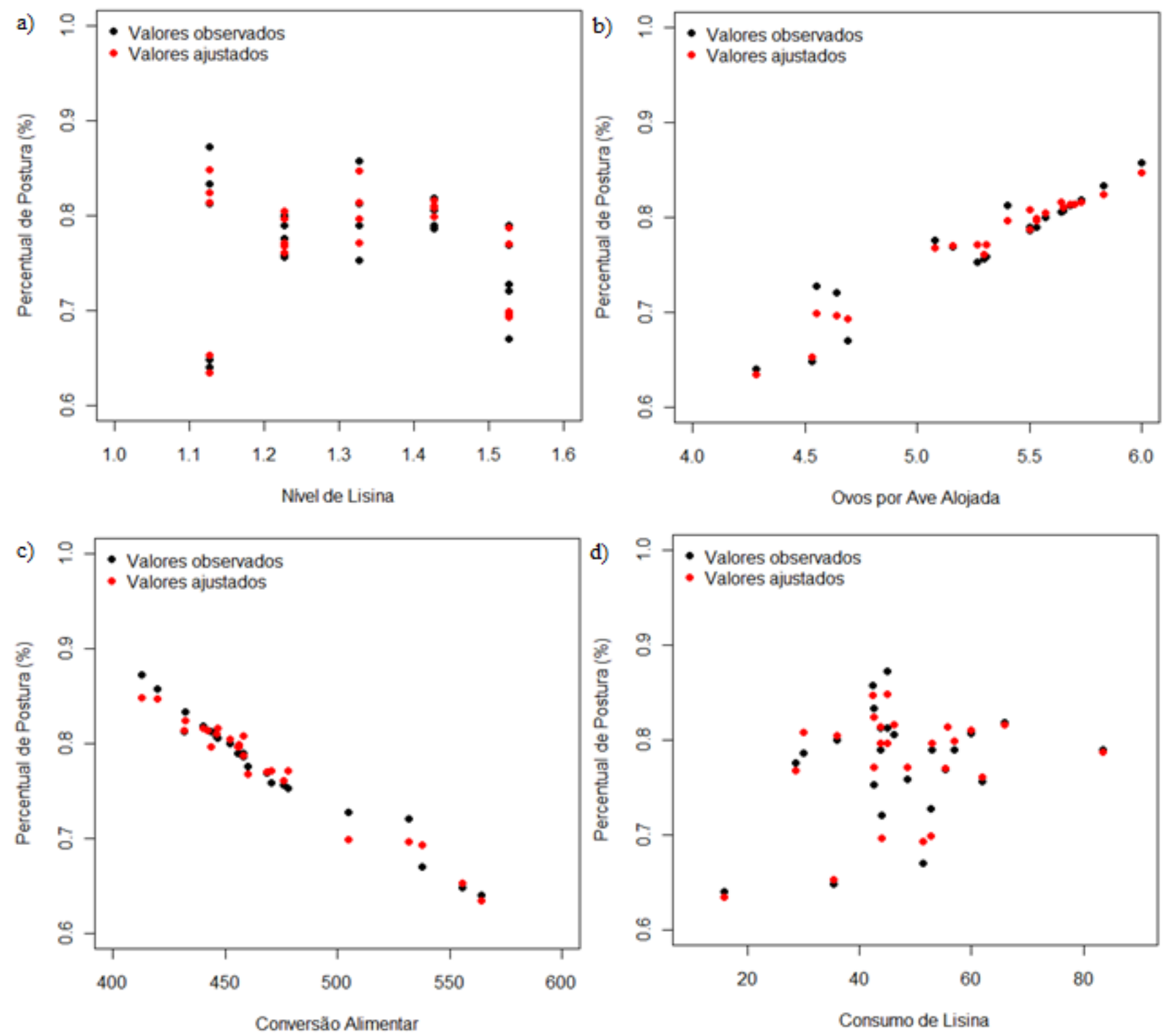

Figura 1. Relação do percentual de postura e os valores ajustados pelo modelo em função das variáveis explicativas: nível de lisina (a), ovos por aves alojadas (b), conversão alimentar (c) e consumo de lisina (d) (Ratio of laying percentage and the values adjusted by model in function of the explanatory variables: lysine levels (a), eggs by housed birds (b), food conversion (c) and lysine consumption (d).

ticos com poder de explicação de $93 \%$ para codornas europeias alimentadas com proteína bruta de $20 \%$ e nível ótimo de lisina de $1,07 \%$, e $54 \%$ quando as codornas foram alimentadas com proteína bruta de $23 \%$ quando o nível ótimo de lisina foi de 1,15\%.

Utilizando modelo de regressão quadrático vários autores explicaram o percentual de postura em codornas japonesas com poder de explicação variando de 25,0\% observado por Moura et al. (2008) com o fornecimento de $1,10 \%$ lisina na dieta; $75,0 \%$ verificado por Lima et al. (2016) com 1,18\% de lisina; $83,0 \%$ obtido por Oliveira et al. (1999) com $1,07 \%$ de lisina; $84,0 \%$ encontrado por Pinto et al. (2003) para nível ótimo de lisina de 1,045; 92,4\% por Nery et al. (2015) com 1,08\% de lisina; até 96,0\% obtido por Costa et al. (2008) quando forneceram $1,03 \%$ de lisina digestível em dietas de codornas japonesas. Isto é decorrente, pois todos os autores utilizaram modelos onde a variável resposta não se restringe ao intervalo $(0,1)$, o que não é o caso do percentual de postura que é uma variável que assume valores entre $(0,1)$ ou 0 a $100 \%$.

\section{CONCLUSÕES}

O percentual de postura das codornas pode ser explicado conjuntamente pelo nível de lisina na dieta, número de ovos por aves alojadas, conversão alimentar e consumo de ração fazendo uso do modelo de regressão beta.

O modelo de regressão beta se mostrou bastante eficaz quando comparado aos modelos empregados usualmente na modelagem do percentual de postura das codornas Europeias. 


\section{BIBLIOGRAFIA}

Barreto, SLT, Araujo, MS, Umigi, RT, Donzele, JL, Rocha, TC, Pinheiro, SRF, Teixeira, RB, Abreu, FVS \& Silva, RF 2006, `Exigência nutricional de lisina para codornas europeias machos de 21 a 49 dias de idade', Revista Brasileira de Zootecnia, vol.35, no.3, pp.750-753.

Costa, FGP, Rodrigues, VP, Goulart, CC, Neto, RCL, Souza, JG \& Silva, JHV 2008, 'Exigências de lisina digestível para codornas japonesas na fase de postura', Revista Brasileira de Zootecnia, vol.37, no.12, pp.2136-2140.

Della-Flora, RP, Germano, JM, Bavaresco, C, Lacerda, V \& Dionello, NJL 2012, 'Exigência nutricional de lisina para matrizes de codornas de corte', Publicação de Medicina Veterinária e Zootecnia, vol.6, no.29, pp.1-15

Ekleston, NI, Madsen, L, Hagar, JC \& Temesgen, H 2011, 'Estimating riparian understory vegetation cover with beta regression and copula models', Forest Science, vol.57, no.3,pp.212-221

Ferrari, SLP \& Cribari-Neto, F 2004, 'Beta regression for modelling rates and proportions', Journal of Applied Statistics, vol.31, no.7, pp.799-815.

Korhonen, L, Korhonen, KT, Stenberg, P, Maltamo, M \& Rautiainen, $M$ 2007, 'Local models for forest canopy cover with beta regression', Silva Fennica, vol.41, no.4, pp.671-685

Laliberte, E, Adair, EC \& Hobbie, SE 2012, 'Estimating litter decomposition rate in single-pool models using nonlinear beta regression', PLOS ONE, vol.7: e45140.

Li, F, Zhang, L \& Davis, CJ 2002, 'Modeling the joint distribution of tree diameters and heights by bivariate generalized beta distribution', Forest Science, vol.48, no. 1, pp.47-58.

Lima, HJD, Barreto, SLT, Donzele, JL, Souza, GS, Almeida, RL, Tinoco, IFF \& Albino, LFT 2016, 'Digestible lysine requirement for growing Japanese quails', The Journal of Applied Poultry Research, vol.25, no.4, pp.483-491.

Lucena, LRR 2016, 'Utilização da lacunaridade na caracterização do vento em Serra Talhada- PE', Biomatemática, vol.26, no.1, pp.53-64.

McCullagh, P \& Nelder, J 1989, 'Generalized linear models', 2nd edition. Chapman and Hall.

Móri, C, Garcia, EA, Pavan, AC, Piccinin, A \& Pizzolante, CC 2005, 'Desempenho e rendimento de carcaça de quatro grupos genéticos de codornas para produção de carne', Revista Brasileira de Zootecnia, vol.34, no.3, pp. 870-876.

Moura, AMA, Soares, RTRN, Nery, VLH, Couto, HP, Fonseca, JB \& Vieira, RAM 2008,' Exigencia de lisina para codornices japonesas (coturnix japonica) durante la puesta', Archivos de Zootecnia, vol.57, no. 220 , pp.439-448.
Nery, HVL, Castro, LG \& Novoa, DMT 2015, 'Digestible lys ine levels for japanese quail in laying phase', Revista de la Facultad de Medicina Veterinaria y de Zootecnia, vol.62, no.3, pp.49-57.

National Research Council - NRC 1994, 'Nutrient requirements of poultry', 9 ${ }^{\mathrm{a}} \mathrm{ed}$, National Academy Press, Washington, D.C.

Oliveira, AM, Furlan, AC, Murakami, AE, Moreira, I, Scapinello, C \& Martins, EN 1999, 'Exigência Nutricional de Lisina para Codornas Japonesas (Coturnix coturnix japonica) em Postura', Revista Brasileira de Zootecnia, vol.28, no.5, pp.1050-1053

Pinto, R, Ferreira, AS, Donzele, JL, Silva, MA, Soares, RTRN, Custódio, GS \& Pena, KS 2003, 'Exigência de Lisina para Codornas Japonesas em Postura', Revista Brasileira de Zootecnia, vol.32, no.5, pp. $1182-1189$.

Quintero, HE, Durland, E, Davis, DA \& Dumham, R 2011 , 'Effect of lipid supplementation on reproductive performance of female channel catfish, Ictalurus punctatus, induced and strip-spawned for hybridization', Aquaculture Nutrition, vol.17, no.2, pp.117-129.

Ribeiro, CLN, Barreto, SLT, Reis, RS, Muniz, JCL, Donzele, JL, Gomes, PC, Junior, JGV \& Albino, LFT 2013, 'Digestible lysine levels in diets for laying Japanese quails', Revista Brasileira de Zootecnia, vol.42, no.7, pp.489-495.

Ribeiro, MLG, Silva, JHV, Dantas, MO, Costa, FGP, Oliveira, SF, Filho, JJ \& Silva, EL 2003, 'Exigências Nutricionais de Lisina para Codornas durante a Fase de Postura, em Função do Nível de Proteína da Ração', Revista Brasileira de Zootecnia, vol.32, no.1, pp.156-161.

Rostagno, HS 2017, 'Tabelas brasileiras para aves e suínos: composição de alimentos e exigências nutricionais', $4^{\mathrm{a}}$ ed, ISE-MG, Viçosa.

Schlegel, P, Havenhand, JN, Gillings, MR \& Williamson, JE 2012, 'Individual variability in reproductive success determines winners and losers under ocean acidification: A case study with sea urchins', PLOS ONE, vol.7: e53118.

Xavier, IMC, Cançado, SV, Figueiredo, TC, Lara, LC, Lana, AMQ, Souza, MR \& Baião, NC 2008, 'Qualidade de ovos de consumo submetidos a diferentes condições de armazenamento', Arquivo Brasileiro de Medicina Veterinária e Zootecnia, vol.60, no.4, pp.953-959. 